\title{
PC Based Optical Salinity Sensor for Different Temperatures
}

\author{
Savarimuthu ROBINSON and Rangaswamy NAKKEERAN
}

Department of Electronics and Communication Engineering, Pondicherry Engineering College, Puducherry-605 014, India

*Corresponding author: Savarimuthu ROBINSON＿E-mail: mail2robinson@pec.edu

\begin{abstract}
The homogeneous, intensity modulated salinity sensor using the photonic crystal ring resonator (PCRR) is proposed and designed for monitoring the salinity of the seawater from $0 \%$ to $100 \%(0 \mathrm{~g} / \mathrm{L}$ to $100 \mathrm{~g} / \mathrm{L})$ at $25^{\circ} \mathrm{C}$. The concentration of the salinity in the seawater changes the refractive index of the seawater. The change in the refractive index of the seawater brings the change in the output signal intensity of the sensor as the seawater flows inside the sensor. By detecting the output power and mapping the salinity level, the salinity can be evaluated. The proposed sensor is composed of periodic Si rods embedded in an air host with a circular PCRR placed between the inline quasi waveguides. Approximately, $2.69 \%$ of output power reduction is observed for every $5 \%$ $(5 \mathrm{~g} / \mathrm{L})$ increase in the salinity as the seawater has a unique refractive index for each salt level. With this underlying principle, the performance of the sensor is analyzed for different temperatures.
\end{abstract}

Keywords: Optical sensor, salinity measurement, photonic crystal, refractive index

\section{Introduction}

Salinity measurement is very important and critical for many industrial fields, such as marine environment monitoring, seasonal climate prediction, military engineering, solar engineering, fishing, offshore oil exploration [1]. Further, it is also commonly used to measure the density of the seawater. Therefore, the research on the salinity measurement is one of the areas which are attracting much attention of researchers.

In the past, several techniques were proposed for determining the salinity. In general, the salinity can be measured either by physical methods or by chemical methods [2]. In physical methods, people use conductivity, density and refractivity phenomena for measurements, whereas in chemical methods people determine sodium chloride $(\mathrm{NaCl})$, and magnesium $(\mathrm{Mg})$ concentration presented in the seawater where the concentration of $\mathrm{NaCl}$ is related to the salinity [2]. Physical methods are quicker and more convenient. Since physical methods are associated with electrical measurements, they can also be affected by electrical interference. This problem could be avoided by using optical techniques for determining and monitoring the salinity of the seawater.

Generally, with optical sensors for the salinity measurement, the refractive index of the seawater is considered here, and the high sensitive refractive index dependent sensor is required to determine the salinity. Recently, fiber optic sensors have attracted the keen attention due to their unique properties such as immunity to electromagnetic interference, high sensitivity, small sensing unit, safety in hazards or explosive environments, the possibility of processing the signal at large distances and the ability to work under high temperature, high voltage

Received: 14 December 2011 / Revised version: 8 February 2012

(C) The Author(s) 2012.This article is published with open access at Springerlink.com 
and high pressure conditions [3, 4].

For sensing the salinity of the seawater, so far, the researchers have been using fiber optic cable [5], fiber optic array [1], surface plasmon resonance (SPR) [6], fiber Bragg grating (FBG) [7] and Fabry-Perot interferometer [8] etc., on the basis of beam deviation, refraction, density and composite variation due to the change in the refractive index. In the reported salinity sensor based on the intensity, the normalized detected output power was low for the input power of $1 \mathrm{~mW}$. Hence, the attempt was made here to enhance the intensity (output power), $Q$ factor and also to reduce the size of the sensor using the photonic crystal ring resonator (PCRR).

Photonic crystals (PCs) are periodic nanostructure or artificial materials in which a periodic variation of the material dielectric constant (refractive index) results in a photonic band gap (PBG). By introducing the defects, it is possible to localize the light in the PBG region [9]. The PC provides a good solution to enable extremely small ring resonator with ultra low bending loss and propagation loss owing to the excellent light confinement [10]. In the literature, PC/PCRR based sensors were reported for chemical [11], biosensing [12], force, strain and pressure sensing applications [13-16], civil, geotechnical [17], also for aqueous environment [18] and underwater acoustic measurement [19] etc.

In this paper, a sensor for sensing the salinity of the seawater is proposed and designed based on two dimensional (2D) circular PCRR. The capability of sensing the salinity of the sensor from $0 \%$ to $100 \%$ $(0 \mathrm{~g} / \mathrm{L}$ to $100 \mathrm{~g} / \mathrm{L})$ is theoretically examined at the temperature of $25{ }^{\circ} \mathrm{C}$. Finally, the variation of the output power with respect to the salinity at different temperatures is also discussed.

\section{Sensing principle}

At the constant temperature, each level of the salinity of the seawater has a unique associated refractive index. It increases by $0.00092 \%$ for every $5 \%(5 \mathrm{~g} / \mathrm{L})$ increase in the salinity [20]. Owing to the variation in the refractive index $(0.00092 \%)$, the output power level $(2.69 \%)$ in inline quasi waveguide based sensor accordingly changes. With the above inherent principle, the salinity of the seawater can be determined by knowing the value of the output power.

The sensor consists of Si pillars in an air host which is packaged in a fluidic channel. The seawater flow via the structure, and the salinity in the seawater alters the refractive index of the sensor which in turn changes the output power. By measuring the output power, it is possible for assessing the presence as well as for quantifying the level of the salinity in the seawater at a chosen temperature.

\section{Photonic crystal ring resonator}

A perfect square lattice of $21 \times 21 \mathrm{PC}$ structure is considered for designing the sensor. The radius of the $\operatorname{rod}$ is $0.185 \times a(100 \mathrm{~nm})$, where " $a$ " is the distance between any two nearest rods, called as the lattice constant. The dielectric constant of the rod is 11.9716 (refractive index, $n$, is 3.46), and the lattice constant is selected to be $540 \mathrm{~nm}$. The structure has a transverse magnetic (TM) photonic band gap over the wavelength range lying between $1241 \mathrm{~nm}$ and $830 \mathrm{~nm}$

Figure 1 depicts the schematic diagram of the seawater sensing with a PCRR based sensor. The transmitter emits the optical signal over a wavelength of interest to the PC based sensor which is placed inside the seawater. The refractive index variation of the seawater with respect to the salinity determines the output intensity available at the sensor output. This in turn is sent to the receiver that converts the optical signal to the electrical signal. The signal processing units map the sensed quantity in the readable form with the help of look-up table, which is displayed in the display board.

The sensor consists of two inline quasi waveguides in horizontal $(\Gamma-x)$ direction and a circular PCRR between them (Fig. 1). The coupling rod is placed either in the input side or in the output 
side of inline quasi waveguides which is denoted as " $i$ ". The structure to be used here for salinity sensing was already analyzed by the authors with a bandpass filter [21]. The arrangement of the inner rod, outer rod and other rods presented in the structure was discussed in [22].

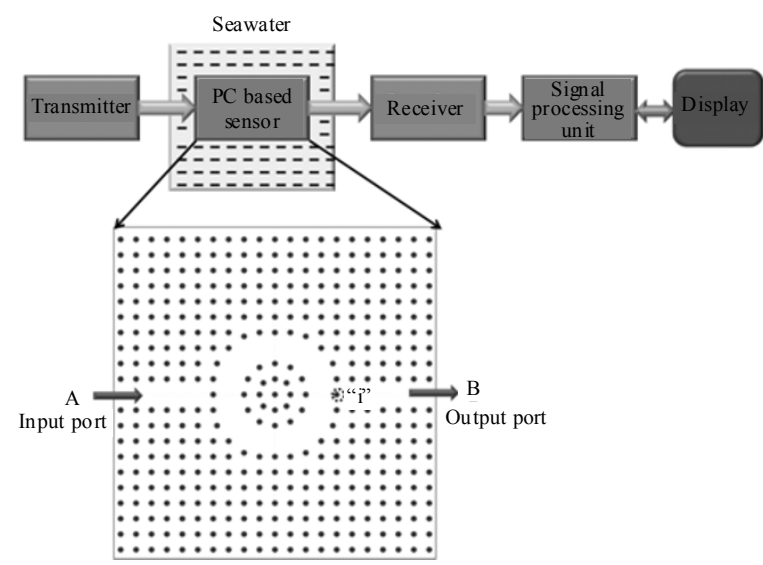

Fig. 1 Schematic structure of the PCRR based sensor for seawater sensing.

To identify the optimum number of coupling rods required to be kept in the inline waveguides for designing the sensor, there are seven different cases considered. The resonant wavelength, $Q$ factor and the output efficiency of those structures with different numbers of coupling rods are listed in Table 1. In Table 1, the resonant wavelength, output efficiency (intensity) and $Q$ factor of the sensor structure with one-one coupling rod are $1590.5 \mathrm{~nm}$, $99.5 \%$ and 477.83 , respectively, where one-one indicates that the number of coupling rods in the input side and output side is one, whereas two-one dictates that the numbers of coupling rods in the input side and output side are two and one, respectively. Similarly, two-one (with $2 a$ ) represents the number of coupling rods in the input side and output side are two and one, respectively, where " $2 \mathrm{a}$ " represents the distance between the two input coupling rods from the cavity. It is seen that an increase in the number of coupling rods sufficiently reduces the output power at the resonant wavelength. However, the change in $Q$ factor and the shift in the resonant wavelength are also noticed which are trivial in nature.
Generally, in the waveguide based ring resonator, while increasing the number of coupling rods, the $Q$ factor increases with the reduced output power [10]. However, in the inline waveguide based ring resonator, there is no significant variation in $Q$ factor while increasing the number of coupling rods in the inline quasi waveguides. Hence, the structure with two coupling rods (one at the input port and the other at the output port) is considered for analyzing the characteristics of the sensor as it offers high output power $(99.5 \%)$ with the sufficient $Q$ factor (478).

Table 1 Resonant wavelength, output efficiency, and $Q$ factor of the structure.

\begin{tabular}{cccc}
\hline $\begin{array}{c}\text { Structures with number of } \\
\text { coupling rods }\end{array}$ & $\begin{array}{c}\text { Resonant wavelength } \\
(\mathrm{nm})\end{array}$ & $\begin{array}{c}\text { Output efficiency } \\
(\%)\end{array}$ & $Q$ factor \\
\hline One-one & 1590.5 & 99.5 & 477.83 \\
Two-one & 1586.5 & 29 & 466.66 \\
Two-one (with 2a) & 1589.0 & 5.2 & 496.56 \\
Two-two & 1589.5 & 23 & 407.56 \\
Three-one & 1590.0 & 0.29 & 441.66 \\
Three-two & 1586.0 & 0.081 & 422.93 \\
Three-three & 1589.5 & 0.067 & 400.37 \\
\hline
\end{tabular}

\section{Evaluation of sensing characteristics}

A temporal light pulse with the input power of $1 \mathrm{~mW}$ is launched into the input port (A) of the inline quasi waveguide. The output signal is recorded by the power monitor at the output port (B). The output power is obtained by applying the fast-Fourier transform (FFT) to the temporal signal recorded by the power monitor. The output signal power from the power monitor kept at the output port is normalized by the input signal power i.e., the output power is the normalized output power.

The normalized output power with respect to the wavelength at the salinity of $0 \%$ at the temperature of $25{ }^{\circ} \mathrm{C}$ is shown in Fig. 2(a). Figure 2(b) shows the variation in normalized output spectra of the sensor for the salinity ranging from $0 \%$ to $100 \%$ at the temperature of $25{ }^{\circ} \mathrm{C}$. The resonant wavelength, $Q$ 
factor and the normalized output power of the sensor at the salinity of $0 \%$ are $1590.55 \mathrm{~nm}, 477.83$, and 99.5\%, respectively. Approximately, 2.69\% reduction in the output power for every $5 \%(5 \mathrm{~g} / \mathrm{L})$ increase in the salinity and $20 \mathrm{~nm}-40 \mathrm{pm}$ shift in the resonant wavelength are observed. As the proposed sensor is the intensity type, the resonant wavelength shift is constant $(20 \mathrm{pm}-40 \mathrm{pm}$ shift per $5 \%$ increase in the salinity is very small). The observed output power of the sensor is higher than the reported one. The observed resonant wavelength shift while changing the salinity from $0 \%$ to $100 \%$ is around $0.5 \mathrm{~nm}$. As the resonant wavelength shift is small (about $1 \mathrm{~nm}$ ), the proposed sensor operates within the detection range. Furthermore, the change in the output power for various refractive indices is highly sufficient to access the salinity of the seawater for a quite large range.

The minimum detectable sensitivity of the proposed sensor is calculated by varying the refractive index [20] for every $0.25 \%, 0.5 \%, 0.75 \%$ and $1 \%$ increase in the salinity at the constant temperature. The detectable intensity variation $(0.55 \%)$ is noticed at the salinity variation of $1 \%$; hence, the sensitivity of the sensor becomes $1 \%$ $(1 \mathrm{~g} / \mathrm{L})$.

Figure 2(c) shows the output power variation with respect to the salinity along with the refractive index. It reveals that the refractive index $(0.00092 \%)$ of the seawater increases while the salinity (5\%) increases, and the normalized output power decreases linearly.

Figure 3 depicts the output power spectra for different temperatures $\left(0{ }^{\circ} \mathrm{C}-25{ }^{\circ} \mathrm{C}\right)$. A $0.5 \%$ increase of the output power is observed for every increase in temperature of $5{ }^{\circ} \mathrm{C}$. The output power variation for different temperatures $\left(0{ }^{\circ} \mathrm{C}-25{ }^{\circ} \mathrm{C}\right)$ with respect to the salinity in the range from $0 \%$ to $40 \%$ is shown in Fig. 4. It clearly shows that the normalized output power decreases as the salinity of the seawater increases. However, it is also observed that the output power increases as the temperature of the seawater increases.

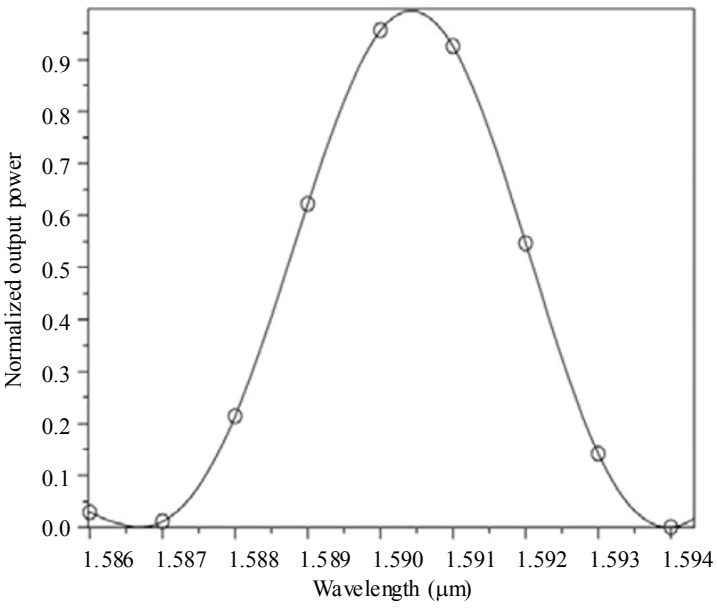

(a)

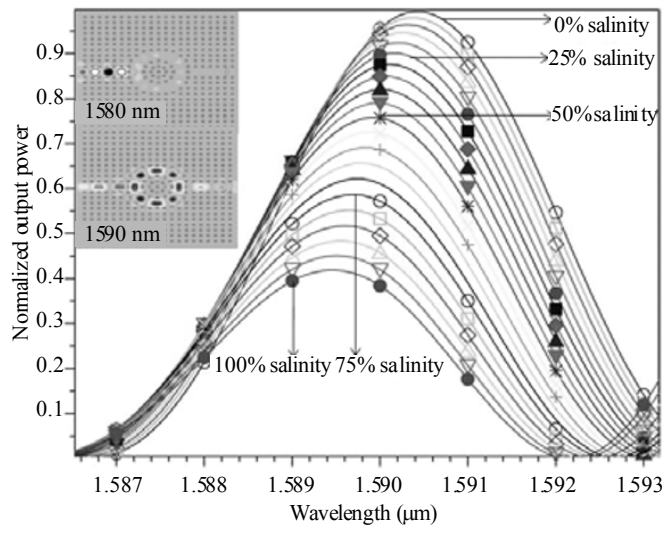

(b)

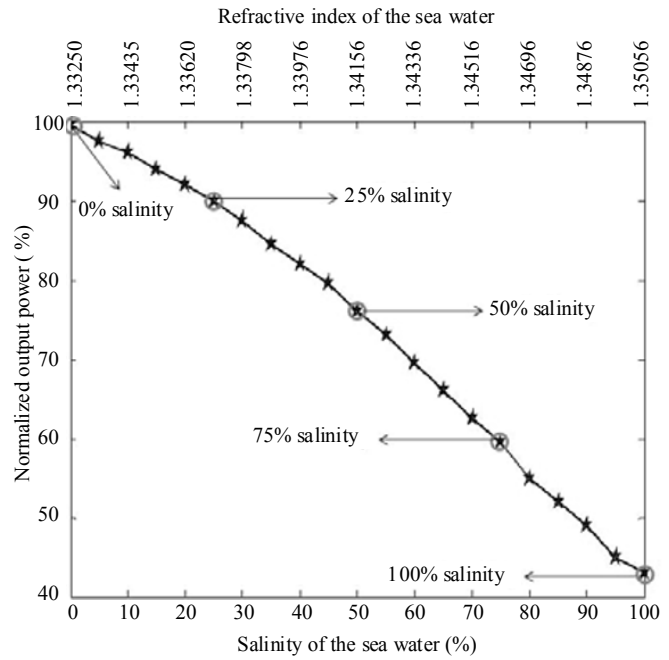

(c)

Fig. 2 Variation in (a) the normalized output power of the sensor for the salinity of $0 \%$ at the temperature of $25^{\circ} \mathrm{C}$, (b) the normalized output power of the sensor for different salt levels at the temperature of $25^{\circ} \mathrm{C}$, and (c) the normalized output power for various salinities $(0 \%-100 \%)$ and their corresponding refractive indices. 


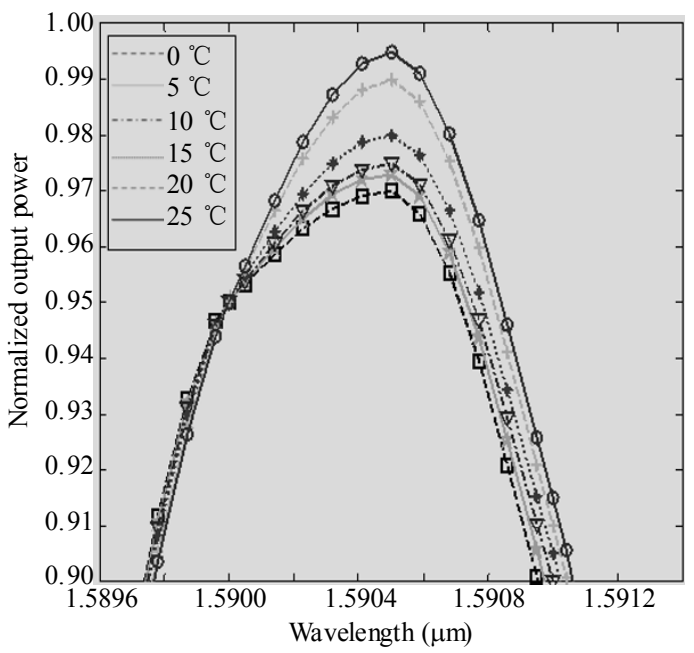

Fig. 3 Normalized output power of the sensor at different temperatures $\left(0{ }^{\circ} \mathrm{C}-25^{\circ} \mathrm{C}\right)$.

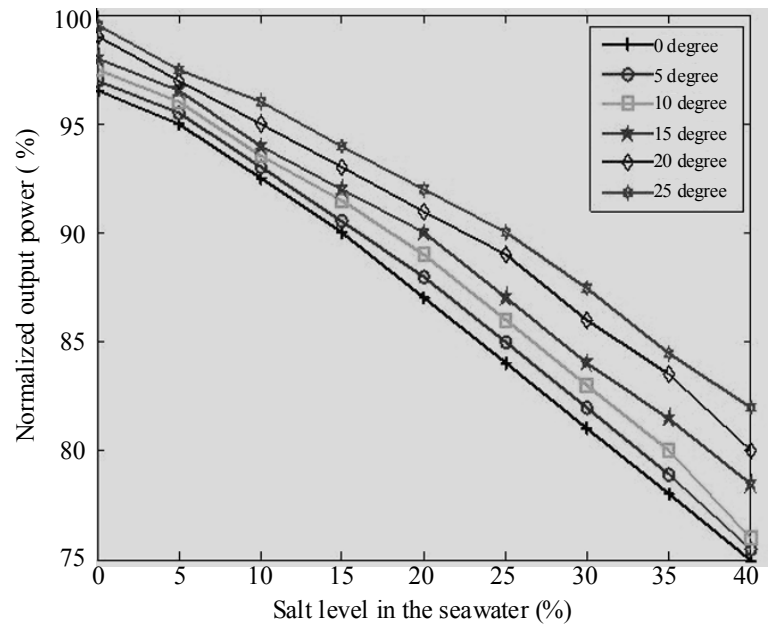

Fig. 4 Variation in the output power with respect to the salinity of the seawater at different temperatures.

The intensity of the proposed sensor is also sensitive to the temperature. Though the temperature of the seawater is changed, it is possible to use it for the salinity detection in the temperature range of the seawater between $0{ }^{\circ} \mathrm{C}$ and $25{ }^{\circ} \mathrm{C}$. In addition, the fluctuation of the optical transmission is critical to the real time environmental monitoring. However, it can be minimized by choosing the prober fabrication and packaging techniques in the near future.

\section{Conclusions}

A sensor is proposed and designed for sensing the salinity of the seawater using two dimensional photonic crystal based circular ring resonator. At the temperature of $25{ }^{\circ} \mathrm{C}$, the observed resonant wavelength, quality factor and output power of the sensor are $1590.55 \mathrm{~nm}, 477.83$, and $99.5 \%$, respectively. It is noticed that there is $2.69 \%$ output power reduction for every $5 \%$ increase in the salinity which is highly sufficient for measuring the salinity of the seawater. The minimum detectable sensitivity and dynamic range of the proposed salinity sensor are $1 \%(1 \mathrm{~g} / \mathrm{L})$ and $100 \%$, and the relevant temperatures are $5{ }^{\circ} \mathrm{C}$ and $25{ }^{\circ} \mathrm{C}$, respectively. Further, the change in the output powers of different temperatures with various salinity levels is also computed. This type of sensors could be deployed for the real time environmental monitoring.

Open Access This article is distributed under the terms of the Creative Commons Attribution License which permits any use, distribution, and reproduction in any medium, provided the original author(s) and source are credited.

\section{References}

[1] Y. Zhao, Y. B. Liao, B. Zhang, and S. R. Lai, "Monitoring technology of salinity in water with optical fiber sensor," IEEE Journal of Lightwave Technology, vol. 21, no. 5, pp. 334-1338, 2003.

[2] S. I. Karaaslan and A. B. Tugrul, "New approach to salinity determination and salinity dispersion along bosphorus," in Eighth International Water Technology Conference (IWTC8 2004), Alexandria, Egypt, pp. 163-173, 2004.

[3] Y. Zhao, P. S. Li, C. S. Wang, and Z. B. Pu, "A novel fiber-optic sensor used for small internal curved surface measurement," Sensors and Actuators A, vol. 86, no. 3, pp. 211-215, 2000.

[4] Y. Zhao, P. S. Li, and Z. B. Pu, "Shape measurement based on fiber-optic technique for complex internal surface," Measurement, vol. 30, no. 4, pp. 289-295, 2001.

[5] N. Díaz-Herrera, O. Esteban, M. C. Navarrete, M. Le Haitre, and A. González-Cano, "In situ salinity measurements in seawater with a fibre-optic probe," Measurement Science and Technology, vol. 17, no. 8, pp. 2227-2232, 2006. 
[6] Y. Zhao, X. Y. Zhang, T. T. Zhao, B. Yuan, and S. Zhang, "Optical salinity sensor system based on fiber-optic array," IEEE Sensors Journal, vol. 9, no. 9, pp. 1148-1153, 2009.

[7] X. Shu, B. A. L. Gwandu, Y. Liu, L. Zhang, and I. Bennion, "Sampled fiber Bragg grating for simultaneous refractive index and temperature measurement," Optics Letters, vol. 26, no. 11, pp. 774-776, 2001.

[8] L. V. Nguyen, M. Vasiliev, and K. Alameh, "Three-wave fiber Fabry-Pérot interferometer for simultaneous measurement of temperature and water salinity of seawater," IEEE Photonics Technology Letters, vol. 23, no. 7, pp. 450-452, 2011.

[9] J. D. Joannopoulos, R. D. Meade, and J. N. Winn, Photonic crystal: modeling of flow of light, Princeton: Princeton Uuniversity Press, 1995.

[10] Z. Qiang, W. Zhou, and R. A. Soref, "Optical add-drop filters based on photonic crystal ring resonators," Optics Express, vol. 15, no. 4, pp. 1823-1831, 2007.

[11] F. L. Hsiao and C. Lee, "Computational study of photonic crystals nano-ring resonator for biochemical sensing," IEEE Sensors Journal, vol. 10, no. 7, pp. 1185-1191, 2010.

[12] D. Dorfnera, T. Zabel, T. Hürlimanna, N. Haukea, L. Frandsenb, U. Ranta, G. Abstreitera, and J. Finleya, "Photonic crystal nanostructures for optical biosensing applications," Biosensors and Bioelectronics, vol. 24, no. 12, pp. 3688-3692, 2009.

[13] T. T. Mai, F. L. Hsiao, C. Lee, W. F. Xiang, C. C. Chen, "Optimization and comparison of photonic crystal resonators for silicon microcantilever sensors," Sensors and Actuators A: Physical, vol.
165, no. 1, pp. 16-25, 2011.

[14] R. M. Silva, M. S. Ferreira, J. L. Santos, and O. Frazao, "Nanostrain measurement using chirped bragg grating fabry-perot interferometer," Photonic Sensors, vol. 2, no. 1, pp. 77-80, 2012.

[15] Q. Yu and X. Zhou, "Pressure sensor based on the fiber optic extrinsic Fabry-Perot interferometer," Photonic Sensors, vol. 1, no. 1, pp. 72-83, 2011.

[16] S. Olyaee and A. A. Dehghani, "High resolution and wide dynamic range pressure sensor based on two-dimensional photonic crystal," Photonic Sensors, vol. 2, no. 1, pp. 92-96, 2012.

[17] W. R. Habel and K. Krebber, "Fiber-optic sensor applications in civil and geotechnical engineering," Photonic Sensors, vol. 1, no. 3, pp. 268-280, 2011.

[18] F. Abdel Malek, "Design of a novel left-handed photonic crystal sensor operating in aqueous environment," IEEE Photonics Technology Letters, vol. 23, no. 3, pp. 188-190, 2011.

[19] S. Huang, X. Jin, J. Zhang, Y. Chen, Y. Wang, Z. Zhou, and J. Ni, "An optical fiber hydrophone using equivalent phase shift fiber grating for underwater acoustic measurement," Photonic Sensors, vol. 1, no. 3, pp. 289-294, 2011.

[20] www.nvcc.edu/home/vzabielski/SeawaterChemistry I.pdf.

[21] S. Robinson and R. Nakkeeran, "Investigation on two dimensional photonic crystal resonant cavity based bandpass filter," Optik Optics, vol. 123, no. 5, pp. 451-457, 2012.

[22] S. Robinson and R. Nakkeeran, "Photonic crystal ring resonator based add-drop filter using Hexagonal rods for CWDM systems," Optoelectronics Letters, vol. 7, no. 3, pp. 164-166, 2011. 